\title{
Region-of-interest breast images with the Twente Photoacoustic Mammoscope (PAM)
}

Manohar, Srirang, Vaartjes, Sanne, van Hespen, Johan, Klaase, Joost, van den Engh, Frank, et al.

Srirang Manohar, Sanne E. Vaartjes, Johan G. C. van Hespen, Joost M.

Klaase, Frank M. van den Engh, Andy K. H. The, Wiendelt Steenbergen, Ton G. van Leeuwen, "Region-of-interest breast images with the Twente Photoacoustic Mammoscope (PAM)," Proc. SPIE 6437, Photons Plus Ultrasound: Imaging and Sensing 2007: The Eighth Conference on Biomedical Thermoacoustics, Optoacoustics, and Acousto-optics, 643702 (13 February 2007); doi: 10.1117/12.699995

SPIE. Event: SPIE BiOS, 2007, San Jose, California, United States 


\title{
Region-of-interest breast studies using the Twente Photoacoustic Mammoscope (PAM)
}

\author{
Srirang Manohar, ${ }^{a,}$ Sanne E. Vaartjes,${ }^{a}$ Johan C. G. van Hespen, ${ }^{a}$ Joost M. Klaase,${ }^{b}$ Frank \\ M. van den Engh, ${ }^{c}$ Andy K. H. Thé, ${ }^{c}$ Wiendelt Steenbergen ${ }^{a}{ }^{a}$ and Ton G. van Leeuwen ${ }^{a}$ \\ ${ }^{a}$ Institute for Biomedical Technology (BMTI), Biophysical Engineering Group, Faculty of \\ Applied Physics (TNW), University of Twente, Postbox 217, 7500AE Enschede, The \\ Netherlands \\ ${ }^{b}$ Department of Surgery, Medisch Spectrum Twente, PB 50000, 7500KA Enschede, The \\ Netherlands \\ ${ }^{c}$ Department of Radiology, Medisch Spectrum Twente, PB 50000, 7500KA Enschede, The \\ Netherlands
}

\begin{abstract}
The Twente Photoacoustic Mammoscope (PAM) is based on generating laser-induced ultrasound from absorbing structures in the breast. The heart of the instrument is a flat PVDF based detector matrix comprising 590 active elements. The exciting source is an Nd:YAG laser operating at $1064 \mathrm{~nm}$ with $5 \mathrm{~ns}$ pulses. The instrument is built around a hospital bed. A study protocol was designed to explore the feasibility of using the photoacoustic technique as embodied in PAM to detect cancer in the breasts of patients with suspect/symptomatic breasts. The protocol was approved by a Medical Ethics testing committee and the instrument approved for laser and electrical safety. The protocol was executed at the Medisch Spectrum Twente by using the mammoscope to obtain photoacoustic region-of-interest (ROI) images of the suspect/symptomatic breasts. We report on one case and compare the photoacoustic images obtained with x-ray mammograms and ultrasound images.
\end{abstract}

\section{INTRODUCTION}

X-ray imaging is the most important modality for the early detection and diagnosis of cancers in the breast. Although a contentious issue, there is a considerable body of literature that shows that x-ray mammographic screening reduces mortality (pg. 34 and references in Nass et al., 2001; Nyström et al. , 2002). It does however, have some drawbacks. Notable among these is in the use of ionizing radiation with its associated potential carcinogenic risks especially in the case of screening asymptomatic women. It has a low positive predictive value which results in unnecessary secondary investigations including biopsies. An accusation levelled against the technique is that suspicious findings also lead to aggressive treatments or over-treatments. X-ray mammography also suffers from poor interpretation and reduced accuracy in the case of younger women or women with breasts possessing high amounts of radiodense glandular tissue.

Ultrasonography has an adjunctive but valuable role to play in the diagnostic examination of symptomatic lesions and screening detected abnormalities. Ultrasound is indicated for problems that it is especially expedient for, such as solid mass-cyst differentiation but only rarely for differentiating malignancy from benign growths. Use of the two modalities - x-ray and ultrasound imaging along with clinical examination, a triple-assessment, can lead to high accuracies. However, at this stage, ultrasound imaging cannot be considered in isolation nor as a first investigation.

Magnetic Resonance Imaging is also gradually gaining a niche role as a problem solving technique in certain cases such as with uncertain findings in mammography and sonography; with cancer recurrence examination in subjects with fibrosis owing to breast-conserving surgery; and for evaluation of multifocii and contralateral

\footnotetext{
* Author for correspondence: S.Manohar@utwente.nl
} 
lesions. However, its poor positive predictive value, high expense and consequent inaccessibility, are as yet major drawbacks.

The enormous burden of breast cancer on society and the room for improvements in the current practice of mammography have been the stimuli for, on the one hand, efforts in pushing the current state of the art in detection and diagnosis forward; and on the other hand, the relentless quest for a newer, different modality that can address the limitations of the current technologies. Of the myriad alternative techniques for breast imaging (see for example Nass et $a l^{1}$ ), a promising area is optical imaging, which uses near-infrared (NIR) light as the probe. NIR imaging is simple and low-cost, and since it does not use ionizing radiation, is risk-free. The technique involves the detection of photons that propagate through the breast and (using models based on the nature of photon propagation in tissue), the reconstruction of the optical properties of the illuminated tissue. The tumor is expected to show an optical absorption contrast compared with healthy tissue, due to an increased blood content arising from an enhanced vascularization.

There is a considerable body of evidence to suggest that the neoplastic properties of a tumor such as unregulated replication may be a necessary but not a sufficient condition for a tumor to develop into a malignant, and lethal phenotype. ${ }^{2}$ For a population of such cells, the proliferating cells are balanced by the cells undergoing apoptosis with the result that the tumor cannot grow beyond a certain size and remains in a dormant state. Cell death is due to lack of nutrients and oxygen owing to the dearth of blood vessels commensurate with the pre-cancerous tumor size. However, various environmental and genetic mechanisms can stimulate the production of blood capillaries in the process of angiogenesis. Once this angiogenic switch has been enabled, the tumor can grow rapidly from its in-situ, microscopic, quiescent state into a symptomatic lesion. It has been suggested that the angiogenic phenotype appears after the expression of the malignant phenotype. ${ }^{2}$

Tumor vessels are expected to be functionally and structurally abnormal; they are highly disorganized, tortuous, and leaky. ${ }^{3}$ It is the presence of hemoglobin in this enhanced, abnormal vascularization which provides an endogenous optical absorption contrast compared with healthy tissue to make tumor detection possible with the use of light. Indeed it has been demonstrated in several optical studies ${ }^{4-7}$ that the optical absorption contrast expected in breast tumors vis-à-vis healthy, background tissue is detectable non-invasively using optical imaging. The hemoglobin oxygen saturation of suspicious sites can additionally serve as a criterion for diagnosing malignancy and can be reconstructed by spectroscopic analysis. ${ }^{4,6,8}$

Light propagation in biological tissue is highly scattering, which results in poor resolution for optical breast imaging. One of the approaches that has been implemented to surmount the resolution impediment is a computational approach ${ }^{9,10}$ using continuous waves, but there have been also technological and methodological modifications to the continuous wave case to permit time-domain ${ }^{11,12}$ and frequency-domain measurements. ${ }^{13,14}$ In frequency-domain techniques the incident light intensity is modulated in the range of tens to hundreds of $\mathrm{MHz}$. Measurement of the amplitude and phase of the exiting light, and fitting it to a theoretical framework based on the diffusion approximation of light propagation in tissue, permits inversion of the model to yield the absorption and scattering coefficients of tissue. In time-domain techniques, the light is administered in picosecond pulses, and using time-gating, the early arriving photons are discriminated from the late-arriving photons of the temporally broadened light pulse. The early gate photons would have travelled shorter and straighter paths than the late gate photons; the early photons would possess the optical absorption properties of a tighter interrogated volume than the late photons, permitting a better resolution. Improving spatial resolution and discriminating between absorption and scattering remain the biggest challenges that are faced by optical imaging.

Photoacoustic imaging avails the endogenous absorption contrast exhibited by tumors to NIR light, without having to combat the problems associated with scattering. Optical contrast on the one hand, and lower scattering experienced by ultrasound in tissue on the other hand, are brought together in this hybrid technique. The mechanism of photoacoustic signal generation consists of the following steps: 1) light is selectively absorbed in higher absorbing regions, when the investigated volume is exposed to pulsed laser radiation; 2) fast non-radiative relaxation of excited states takes place with thermalization of absorbed optical energy; 3) the resulting local thermal expansion produces pressure transients. The pressure pulses propagate as ultrasound to the surface where it is detected using wide-frequency band detectors. ${ }^{15,16}$ The time-of-flight, amplitude and duration of the pressure transient generated under irradiation conditions of pressure and thermal confinement* provide informa-

*Pressure and temperature relaxation during time scale of the laser (heating) pulse duration is negligible 


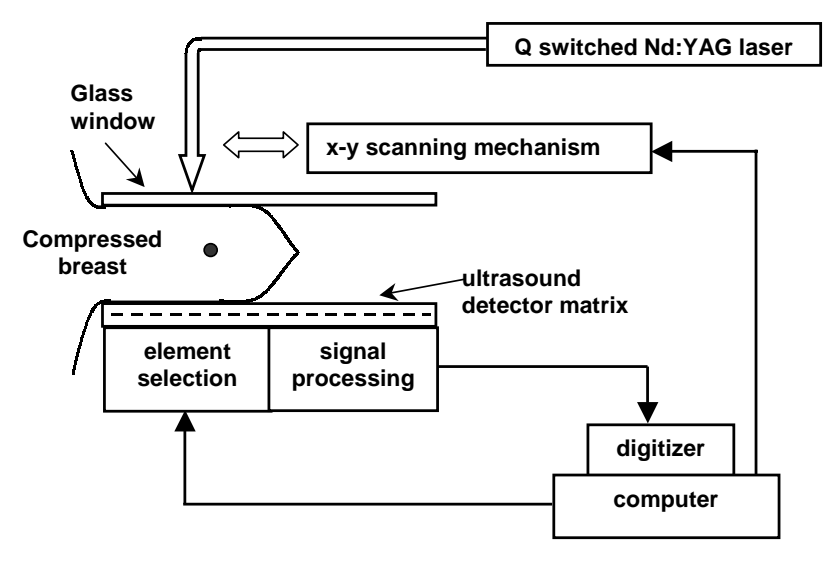

Figure 1: Schematic of the Photoacoustic Mammoscope.

tion regarding location, strength and dimensions of the acoustic source, thereby permitting a reconstruction of the absorber.

The feasibility of using photoacoustics for breast cancer detection has been demonstrated in restricted studies using near-infrared light. ${ }^{17,18}$ There is also an increased ionic water content in the cancer due to the increased concentration of blood and other proteins associated with the cancer. This has been demonstrated using radio waves $(434 \mathrm{MHz})$ in a few studies. ${ }^{19,20}$ However, there have been as yet no large published clinical trials and the technique is as yet in its incipient stages; the original patents being issued as late as $1998 .{ }^{21,22}$

In this article, we present initial results of a pilot study to evaluate the feasibility of the recently developed clinical prototype of a photoacoustic imager for breast imaging. This instrument called the Twente Photoacoustic Mammoscope $(\mathrm{PAM})^{23}$ is based on a multi-element flat ultrasound detector matrix. PAM uses near-infrared (NIR) light at $1064 \mathrm{~nm}$ as the exciting source. We begin by presenting the important characteristics of PAM, discuss the study protocol of the pilot study and finally discuss the result of one case.

\section{THE PHOTOACOUSTIC MAMMOSCOPE (PAM)}

The Twente Photoacoustic Mammoscope uses a planar geometry with a flat ultrasound detector matrix. The geometry requires mild compression of the breast between a transparent glass window and the detector matrix. This is shown in the schematic in Fig. 1. The transparent window is present in a compartment that contains an x-y scanning system which translates the output of a light delivery system (LDS) in 2-dimensions, to locally illuminate the surface of the breast through the glass window. PA generated ultrasound propagates through the breast to be recorded by the ultrasound detector matrix at the opposite side. A number of detectors appropriate to the desired scanning area are read out one-by-one. The control lines of the device are controlled via a digital input-output card in the PC. The data line is fed to 1 channel of a dual channel $100 \mathrm{MHz}, 100 \mathrm{MS} / \mathrm{s}, 8$ bit digitizer (NI-5112, National Instruments, Austin) card in the PC. The PC runs a LABView program that controls the scanning system, the detector array, and digitizer. Three-dimensional images are reconstructed using a delayand-sum beam-focusing algorithm. A volume of $50 \mathrm{~mm}$ x $50 \mathrm{~mm} \times 60 \mathrm{~mm}$ is reconstructed on a $50 \times 50 \times 60$ grid in 2 minutes.

The ultrasound detector matrix consists of 590 elements based on spring loaded contacts to a $110 \mu \mathrm{m}$ PVDF film. The detector is part of the Achilles InSight ${ }^{\mathrm{TM}}$ bone densitometer from General Electric Medical Systems (Lunar) and has been adapted for use in PAM. The electronics available onboard allows the accessing of 1 element at a time. Figure 2(a) shows a photograph of the detector; fig. 2(b) shows the layout of the 590 elements.

The instrument is built into a hospital bed as shown in figure 3(a). The subject lies prone on the bed with her breast pendant through the aperture in the bed. The breast is mildly compressed between the detector matrix 
and the glass plate of the compartment which carries an $x-y$ scanning system. Compression is required to obtain a uniform thickness of the breast and a good acoustic contact with the detector. This is achieved by manually turning the handwheel of the compression mechanism. The scanning system based on lead screws has as payload the output of a light delivery assembly (not shown). The driving mechanism of the scanning system is shown in figure 3(b).

The laser is present at the bottom of the bed and mounted on the frame. To transport the light from the laser to the $\mathrm{x}-\mathrm{y}$ scanning system a mechanism consisting of 2 reflecting prisms arranged in the form of a periscope are used (not shown). More details are provided in Ref.. ${ }^{23}$

Some important specifications of the instrument are consolidated in Table 1; further details including system performance on liquid and solid phantoms may be found elsewhere. ${ }^{23-25}$

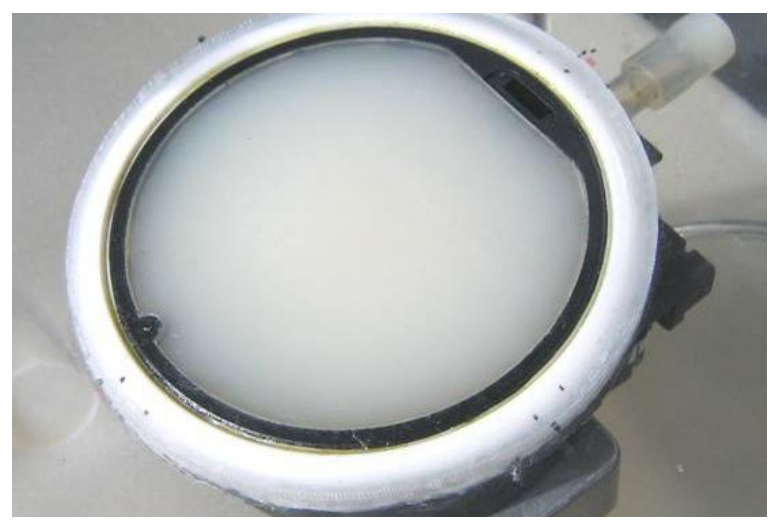

(a)

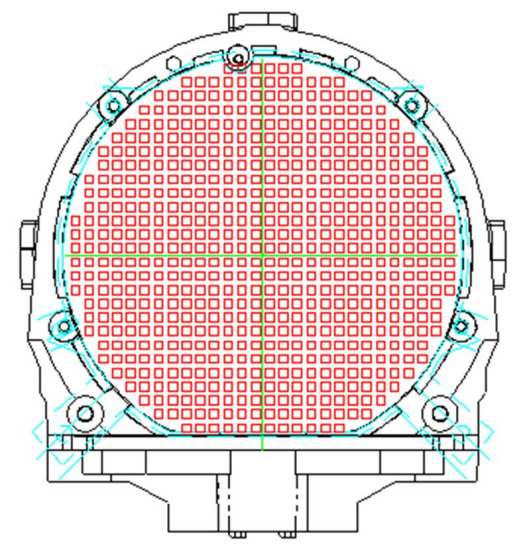

(b)

Figure 2: (a) Photograph of the detector matrix. The device is protected by an $18 \mathrm{~mm}$ thick polymer layer (b) Layout of the 590 elements.

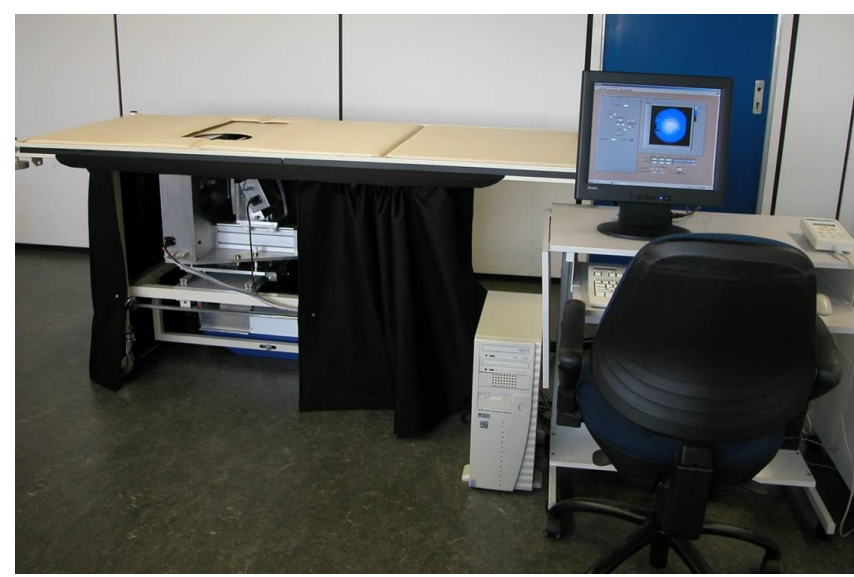

(a)

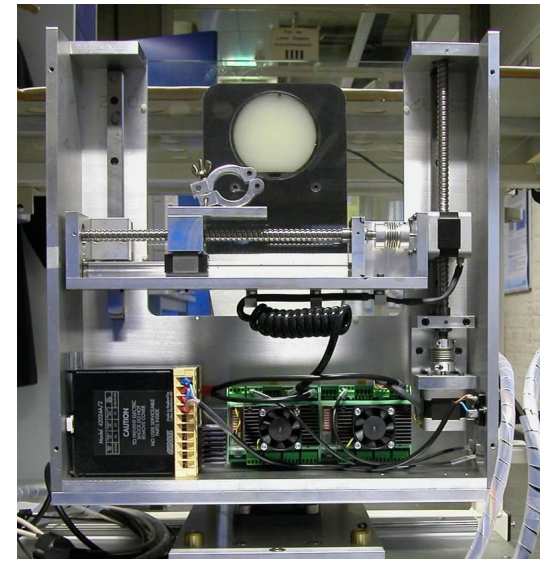

(b)

Figure 3: (a) Photograph of the Photoacoustic Mammoscope (PAM). The instrument is built into a hospital bed. The subject lies prone on the bed with the symptomatic/suspect breast inserted through the aperture into the instrument beneath. (b) A head-on view of the instrument through the glass plate of the scanning system compartment. The detector is seen on the far side. 
Table 1: Specifications of PAM.

\begin{tabular}{lll}
\hline Laser $^{\mathrm{a}}$ & wavelength & $1064 \mathrm{~nm}$ \\
& pulse width & $5 \mathrm{~ns}$ \\
Detector $^{\mathrm{b}}$ & repetition rate & $10 \mathrm{~Hz}$ \\
& matrix size & $90 \mathrm{~mm}$ \\
& number of elements & 590 \\
& element size & $2 \times 2 \mathrm{~mm}$ \\
& central frequency & $1 \mathrm{MHz}$ \\
& bandwidth $(-6 \mathrm{~dB})$ & $130 \%$ \\
Reconstruction & algorithm & delay \& sum \\
& lateral resolution & $3.1-4.4 \mathrm{~mm}$ \\
& axial resolution & \\
\end{tabular}

${ }^{\text {a }}$ Brilliant B, Quantel (Paris)

b Lunar Corporation, General Electric (Wisconsin, Madison)

${ }^{\mathrm{c}}$ For depths between 15 and $60 \mathrm{~mm}$.

\section{IMAGING PROTOCOL}

Using the experiences gained from phantom ${ }^{26,27}$ and healthy volunteer measurements, ${ }^{25}$ a study protocol was formulated ${ }^{28}$ for a pilot phase of clinical trials. For this phase, the aims may be enunciated as follows:

1. Evaluation of the feasibility of the technique as embodied in PAM in detecting breast tumors in symptomatic subjects, in order to corroborate the earlier performances on realistic phantoms.

2. Comparison of clinical, pathological and conventional imaging findings with photoacoustic images, to understand in retrospect the correspondence between the photoacoustic images and morphological, and pathological features of tumors.

3. Effect technological changes based on experiences as above, and carry forward various results to prepare the instrument for a more rigorous evaluation.

In this phase the basic hypothesis of tumor detection, using photoacoustics as embodied in this instrument, will be studied. This phase will employ preferably 10 patients, but up to a maximum of 25 patients, with palpable tumors, which after clinical examinations and diagnostic imaging are suspect for malignancy. This phase will provide valuable information, data and experience in order to optimize the instrument and the experimental protocol for the subsequent phases. In case the outcome of the study satisfies certain success criteria, the experience will be carried forward into further phases of the research. In these it is proposed to carry out studies governed by a separate protocol to attempt a more rigorous evaluation of the technique.

The standard examination comprised a craniocaudal (CC) view of the symptomatic/suspect breast of the subject. The patient lies prone on the bed, with her breast pendant through the aperture in the bed. A clinician, preferably the same who has performed the x-ray mammogram, or ultrasound or MRI examination will be responsible for the positioning of the breast between the illumination compartment and the ultrasound detector, in such as manner that the imaging will be performed principally in the area which harbors the tumor. Mild compression will be applied to the breast with copious amounts of acoustic coupling gel between the breast and the detector with the sole purpose of obtaining a large area, good acoustic contact. The compression is applied gradually, by manually turning a handwheel that linearly drives the carriage holding the detector. At the instant the patient indicates any discomfort, further application of compression is terminated. The patient is then expected to lie immobile in this position for a period up to 45 minutes, during which period of time the scan will be performed.

The protocol was approved by the Medisch Ethische Toetsingscommissie (METC) of the Medisch Spectrum Twente, Enschede, The Netherlands. 

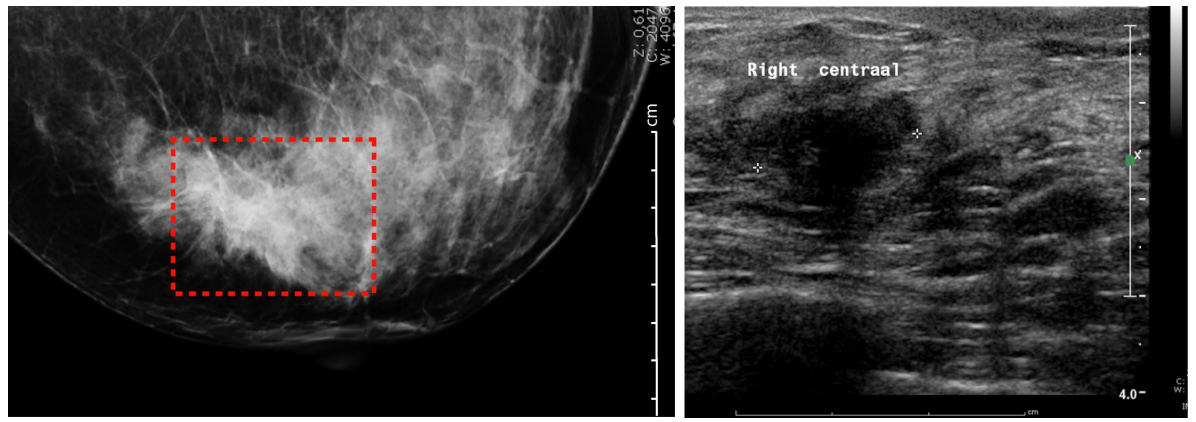

Figure 4: (L) The x-ray mammogram in cranio-caudal projection of a subject with a palpable mass in the right breast. The mammogram shows an area with architectural distortion in a region with microcalcifications. (R) Breast sonogram showing an irregular mass with hypoechogenicity.

\section{RESULTS}

We discuss the results of photoacoustic scanning in the region-of-interest (ROI) of the suspect breast of a 50 year Caucasian woman. A large tumor had been discovered at 11 'o' clock in the right breast by palpation. The x-ray mammogram made using the Selenia Full Field Digital Mammography System (Hologic, Bedford, USA) taken in cranio-caudal projection is shown in Fig. 4 at left. The image reveals an area of architectural distortion with spiculations emanating from a region of microcalcifications. No mass is evident in the image. The dotted line encloses the region-of-interest (ROI) in which we think the photoacoustic scan was performed. However it must be emphasized here that it is not possible to make any point-by-point comparison between the ROI in x-ray mammogram and any photoacoustic image because of difference in the degree of compression between the 2 techniques. Breast ultrasonography used a Philips iU22 ultrasound system (Philips Medical System, Best, The Netherlands) equipped with a L12-5 $50 \mathrm{~mm}$ linear probe operated at $12 \mathrm{MHz}$. The ultrasound image (Fig. 4 at right) shows an irregular hypoechoic mass which is poorly marginated. The size of the lesion is estimated to be

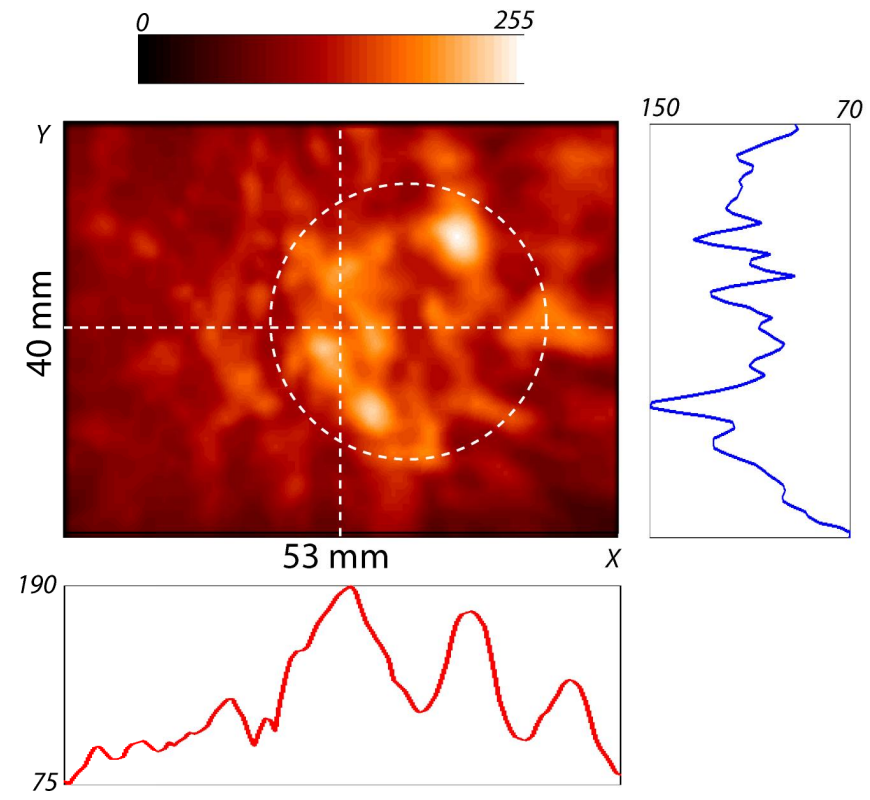

Figure 5: (L) The photoacoustic MIP image in top view showing higher intensity regions. (This image is best viewed using a HSV colormap.) We attribute the higher absorbing regions to tumor vascularization. Also shown are the intensity profiles through the photoacoustic data along lines marked in the MIP image. 
$17 \mathrm{~mm}$.

Based on clinical examination and diagnostic x-ray and ultrasound imaging the lesion was highly suspect for malignancy. Core biopsy was recommended. At this point we performed the photoacoustic scan covering $53 \times 40 \mathrm{~mm}$. The photoacoustic maximum-intensity projection (MIP) image is shown in Fig. 5 (left) in top view. Higher intensity regions are clustered heterogeneously in the region of where the lesion is expected to reside. These are due to regions of higher absorption in the breast. We contend that these hot spots are evidence of vascularization; we are seeing the vascular signature of the cancer. The high intensity regions are observed (not shown in this article) in the photoacoustic images starting at a depth of $9 \mathrm{~mm}$ from the illuminated surface and extending to approximately $18 \mathrm{~mm}$ below the surface. A dotted line is superimposed on the MIP image as a guide to the eye enclosing the vascularity. We believe that this area measuring roughly $30 \mathrm{~mm}$ is an indicator of cancer size. Profiles though the reconstructed data indicate that even though the background level is high as is to be expected in a MIP image, the average image contrast that is observed between the background and cancer vascularity is roughly of the order of $1.5-1.8$.

The dignity of the lesion was confirmed post-surgically to be an invasive ductal carcinoma. The maximum size of the cancer was determined histopathologically to $32 \mathrm{~mm}$.

\section{DISCUSSION}

We have been able to visualize regions of higher intensity in photoacoustic images whose distribution in the suspect region of the breast leads us to believe that the higher absorption arises from the blood content associated with cancer vascularity. Indeed in 4 of the 5 technically acceptable measurements performed so far, high optical absorbing regions are clearly visible in the reconstructions. The size of the cluster enclosing the vascular hot spots in the case described and in the other cases is comparable with the histopathologically estimated cancer sizes.

Also the experiences of patients who participated in the study were largely agreeable. Apart from the inconvenience of remaining immobile in a prone position for more than 30 minutes almost no patient found the experience discomforting or painful.

We believe that the first studies using the Twente Photoacoustic Mammoscope are successful. We have demonstrated the feasibility of photoacoustic imaging as embodied in PAM, in detecting tumors in the breasts of human patients. We will continue the pilot study and attempt to focus on the following aspects:

1. Study minimal detectable cancer size, spatial resolution and maximum imaging depth.

2. Study certain number of benign phenotypes and understand the photoacoustic images in such cases.

3. Define tumor and/or subject characteristics that allow cancer to be detected using photoacoustics. This knowledge will help in judging the niche role that the technique may have to play in future in being applied for certain cancer types and/or in certain subjects.

4. Develop lesion visibility scoring as a means to describe the performance of PAM in detecting cancer.

\section{ACKNOWLEDGMENTS}

S. Manohar is supported in the Vernieuwingsimpuls program (Veni) by the Nederlandse Wetenschappelijk Organisatie (NWO) and Stichting Technische Wetenschappen (STW). The authors acknowledge Dr. H. J. C. M. Sterenborg (Erasmus Medisch Centrum, Rotterdam) for suggestions and Dr. A. Stam (Medisch Spectrum Twente, Enschede) for maintaining patient records. Nursing staff of the Mammacare department of the Medisch Spectrum Twente are acknowledged for cooperation and help during photoacoustic measurements. 


\section{REFERENCES}

1. S. J. Nass, I. C. Henderson, and J. C. Lashof, Mammography and beyond: Developing technologies for the early detection of breast cancer. Report of the institute of medicine, Washington, DC : National Academy Press, March 2001.

2. J. Folkman, Tumour angiogenesis, ch. 9, pp. 132-52. B. C. Decker, Hamilton, 5 ed., 2000.

3. P. Carmeliet and R. K. Jain, "Angiogenesis in cancer and other diseases," Nature 407, pp. 249-57, 2000.

4. B. W. Pogue, S. P. Poplack, T. O. McBride, W. A. Wells, K. S. Osterman, U. L. Osterberg, and K. D. Paulsen, "Quantitative hemoglobin tomography with diffuse near-infrared spectroscopy: Pilot results in the breast," Radiology 218, pp. 261-66, 2001.

5. T. McBride, B. W. Pogue, S. Jiang, U. L. Osterberg, K. D. Paulsen, and S. P. Poplack, "Initial studies of in vivo absorbing and scattering heterogeneity in near-infrared tomographic breast imaging," Opt. Lett. 26, pp. 822-4, 2001.

6. B. J. Tromberg, N. Shah, R. Lanning, A. Cerussi, J. Espinoza, T. Pham, L. Svaasand, and J. Butler, "Non-invasive in vivo characterization of breast tumours using photon migration spectroscopy," Neoplasia 2, pp. 26-40, 2000.

7. K. Suzuki, Y. Yamashita, K. Ohta, M. Kaneko, M. Yoshida, and B. Chance, "Quantitative measurement of optical parameters in normal breasts using time-resolved spectroscopy: In vivo results of 30 Japanese women," J. Biomed. Opt. 1, pp. 330-4, 1996.

8. R. L. P. V. Veen, A. Amelink, M. Menke-Pluymers, C. V. der Pol, and H. J. C. M. Sterenborg, "Optical biopsy of breast tissue using differential path-length spectroscopoy," Phys. Med. Biol. 50, pp. 2573-81, 2005.

9. J. H. Hoogenraad, M. B. van der Mark, S. B. Colak, G. W. 't Hooft, and E. S. van der Linden, "First results from the Philips Optical Mammoscope," in Photon Propagation in Tissues III, D. A. Benaron, B. Chance, and M. Ferrari, eds., Proc. Soc. Photo-Opt. Instrum. Engg. (SPIE) 3194, pp. 184-90, 1997.

10. S. B. Colak, M. B. van der Mark, G. W. 't Hooft, J. H. Hoogenraad, E. S. van der Linden, and F. A. Kuijpers, "Clinical optical tomography and NIR spectroscopy for breast cancer detection," IEEE J. Select. Topics Quantum Electron. 5, pp. 1143-58, 1999.

11. V. Ntziachristos, X. H. Ma, and B. Chance, "Time-correlated single photon counting imager for simultaneous magnetic resonance and near-infrared mammography," Rev. Sci. Instrum. 69, pp. 4221-33, 1998.

12. D. Grosenick, H. Wabnitz, H. H. Rinneberg, K. T. Moesta, and P. M. Schlag, "Development of a time-domain optical mammograph and first in vivo applications," Appl. Opt. 38, pp. 2927-43, 1999.

13. S. Fantini, K. T. Moesta, M. A. Franceschini, H. Jess, H. Erdl, E. Gratton, P. M. Schlag, and M. Kaschke, "Instrumentation and clinical applications in frequency-domain optical mammography," in Proc. 19th Ann. Intl. Conf. IEEE Engg. Med. Biol. Soc., (6), pp. 2741-4, IEEE, IEEE Press, (Piscataway), 1997.

14. B. W. Pogue, M. Testorf, T. McBride, U. Osterberg, and K. Paulsen, "Instrumentation and design of a frequency-domain diffuse optical tomography imager for breast cancer detection," Opt. Express 1, pp. 391403, 1997.

15. C. G. A. Hoelen, A. Dekker, and F. F. M. de Mul, "Detection of photoacoustic transients originating from microstructures in optically diffuse media such as biological tissue," IEEE Trans. Ultrason. Ferroelect. Freq. Contr. 48, pp. 37-47, 2001.

16. V. G. Andreev, A. A. Karabutov, and A. A. Oraevsky, "Detection of ultrawide-band ultrasound pulses in optoacoustic tomography," IEEE Trans. Ultrason. Ferroelectr. Freq. Control 50, pp. 1383-90, 2003.

17. A. A. Oraevsky, E. V. Savateeva, S. V. Solomatin, A. A. Karabutov, V. G. Andreev, Z. Gatalica, T. Khamapirad, and P. M. Henrichs, "Optoacoustic imaging of blood for visualization and diagnostics of breast cancer," in Biomedical Optoacoustics III, A. A. Oraevsky, ed., Proc. Soc. Photo-Opt. Instrum. Engg. (SPIE) 4618, pp. 81-94, 2002.

18. A. Oraevsky, V. G. Andreev, A. A. Karabutov, S. V. Solomatin, E. V. Savateeva, R. D. Fleming, Z. Gatalica, and H. Singh, "Laser optoacoustic imaging of breast cancer in vivo," in Biomedical Optoacoustics II, A. A. Oraevsky, ed., Proc. Soc. Photo-Opt. Instrum. Engg. (SPIE) 4256, pp. 6-15, 2001.

19. R. A. Kruger, W. L. Kiser Jr., D. R. Reinecke, and G. A. Kruger, "Application of thermoacoustic computed tomography to breast imaging," in Physics of Medical Imaging, J. M. Boone and J. T. Dobbins III, eds., Proc. Soc. Photo-Opt. Instrum. Engg. (SPIE) 3659, pp. 426-30, 1999. 
20. R. A. Kruger, K. D. Miller, H. E. Reynolds, W. L. Kiser Jr., D. R. Reinecke, and G. A. Kruger, "Contrast enhancement of breast cancer in vivo using thermoacoustic CT at $434 \mathrm{MHz}$. ." Radiology 216, pp. 279-83, 2000 .

21. A. A. Oraevsky, S. L. Jacques, and R. O. Esenaliev, "Optoacoustic imaging for medical diagnosis," US Patent 5,840,023, 1998.

22. R. A. Kruger, "Photoacoustic breast scanner," US Patent 5,713,356, 1998.

23. S. Manohar, A. Kharine, J. G. C. van Hespen, W. Steenbergen, and A. G. C. van Leeuwen, "The Twente Photoacoustic Mammoscope: system overview and performance," Phys. Med. Biol. 50, pp. 2543-57, 2005.

24. S. Manohar, A. Kharine, J. G. C. van Hespen, W. Steenbergen, and A. G. C. van Leeuwen, "Photoacoustic mammography laboratory prototype: imaging of breast tissue phantoms," J. Biomed. Opt. 9, pp. 1172-81, 2004 .

25. S. Manohar, A. Kharine, C. de Vos, J. C. G. van Hespen, W. Steenbergen, and T. G. van Leeuwen, "The Twente Photoacoustic Mammoscope: Towards clinical trials," in Emerging Technologies in Breast Imaging, J. S. Suri, R. Rangayyan, and S. Laxminarayan, eds., American Scientific Publishers, p. In print, 2007.

26. S. Manohar, A. Kharine, J. G. C. van Hespen, W. Steenbergen, F. F. M. de Mul, and A. G. C. van Leeuwen, "Photoacoustic imaging of embedded inhomogeneities in breast tissue phantoms," in Biomedical Optoacoustics IV, A. A. Oraevsky, ed., Proc. Soc. Photo-Opt. Instrum. Engg. (SPIE) 4960, pp. 64-75, 2003.

27. S. Manohar, A. Kharine, W. Steenbergen, and A. G. C. van Leeuwen, "Three-dimensional photoacoustic imaging of breast tissue phantoms," in International Conference on Advanced Laser Technologies (ALT 03), I. Shcherbakov, ed., Proc. Soc. Photo-Opt. Instrum. Engg. (SPIE) In print, 2003.

28. H. J. C. M. Sterenborg, M. Menke, C. C. M. Bartels, M. M. A. Tilanus-Linthorst, S. Manohar, A. Kharine, and W. Steenbergen, "Pilot study of photoacoustic mammography prototype by examination of patients with symptomatic breast lesions," in Protocol approved by the Medisch Ethische Toetsingscommissie (METC), Medisch Spectrum Twente, Enschede, The Netherlands, 2005. 